\title{
Elastic scattering and total reaction cross sections with low-energy light radioactive ion beams.
}

\author{
Valdir Guimarães ${ }^{1}$ \\ e-mail: v.guimaraes@if.usp.br \\ Instituto de Física, Universidade de São Paulo P.O.Box 66318, 05389-970 São Paulo, SP, Brazil.
}

\begin{abstract}
Elastic scattering experiments have being performed with low-energy radioactive ion beams produced by the RIBRAS facility in Sao Paulo, Brazil. Here I present the results for elastic scattering of ${ }^{6} \mathrm{He}$ on several targets and light beams on ${ }^{12} \mathrm{C}$ target. Special emphasis is given to the analysis of experiments were angular distributions for the elastic scattering of beryllium isotopes projectiles, ${ }^{7} \mathrm{Be},{ }^{9} \mathrm{Be}$ and ${ }^{10} \mathrm{Be}$, on a light target ${ }^{12} \mathrm{C}$ were obtained. These elastic scattering angular distributions have been analysed in terms of optical model using the double-folding Sao Paulo potential. From this analysis, the total reaction cross section were also deduced and compared to the total reaction cross sections for many other light projectiles on ${ }^{12} \mathrm{C}$ target. The comparison was made in terms of Universal Function reduction method.
\end{abstract}

\section{Introduction}

The possibility of using radioactive nuclear beams has opened an exciting $f$ eld of investigation in nuclear physics. Many facilities have installed or upgraded experimental devices and/or developed new techniques to produce intense radioactive nuclear beams. The idea of these facilities is to investigate nuclei at extreme conditions in terms of density, temperature, angular momentum and isospin. Reactions induced by these beams have now being performed and exotic nuclear structure such as halo properties have been investigated.

Although large laboratories are pushing to produce all kinds of exotic and very energetic species of nuclei, some efforts have also been devoted by small laboratories to produce low-energy light radioactive beams. In particular, two facilities, RIBRAS at São Paulo, Brazil [1,2], and Twinsol at Notre Dame, USA [3] have produced light nuclei radioactive beams such as ${ }^{6} \mathrm{He},{ }^{7} \mathrm{Be},{ }^{8} \mathrm{Li},{ }^{8} \mathrm{~B}$ and others. These beams are being used to investigate low-energy reactions such as elastic scattering, transfer, breakup and fusion reactions, which can provide useful information on the structure of light nuclei near the dripline. Reactions induced by radioactive light particles are of interest not only to investigate exotic structure of these nuclei but also for nuclear astrophysics, where they can have important consequences on the abundances of other nuclei in many environments [4]. The investigation of low energy reactions with light exotic nuclei is also of some interest from the theoretical point of view since many of the high energy approximations that simplify the theoretical treatments are no longer valid.

\section{Production of Low-energy Radioactive Nuclear Beams RNB.}

To produce secondary low-energy radioactive ion beams, the University of Notre Dame, USA [3] and later the University of São Paulo, Brazil [1] decided to adopt a system based on superconducting solenoids. The solenoids in these systems, $6 \mathrm{~T}$ and $6.5 \mathrm{~T}$ respectively, act as thick lenses to collect, select, and focus the secondary beam into a scattering chamber. In principle, any type of recoil mass separator could be used to select and deliver secondary beams. However, systems based on solenoids can have the advantage of a large angular acceptance. In the case of the RIBRAS system, the large warm bore $(30 \mathrm{~cm}$ clear) can provide an angular acceptance of about $30 \mathrm{msr}$ in solid angle or $2^{0}<\theta<15^{0}$. An artistic picture view of the RIBRAS system installed at Sao Paulo is shown in Fig. 1. In this system, the production target consists of a gas cell, mounted in a ISO chamber with a $2.2 \mu \mathrm{m}$ Havar entrance window and a ${ }^{9} \mathrm{Be}$ vacuum tight exit window $16 \mu \mathrm{m}$ thick, which plays the role of the primary target and the window of the gas cell. The gas inside the cell has the purpose of cooling the Beryllium foil heated by the primary beam but can also be used as production target. The f rst magnet selects, in-f ight, ions produced in the primary target by its magnetic rigidity, $m E / Q^{2}$. The primary beam is suppressed in a tungsten Faraday cup, which also measures its intensity, while the secondary beam is selected by the solenoids. The purity of the interested secondary radioactive beam is determined by a system of blocking and apertures along the beam line. Also, if we use two solenoids, it is possible to use differential energy loss in an energy degrader foil, located at the crossover point between the magnets, to select the ion of interest and move the contam- 


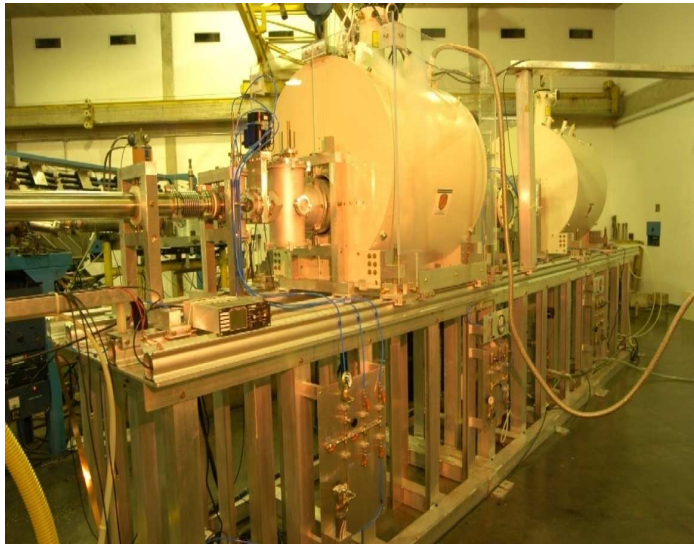

Fig. 1. Picture view of the RIBRAS system at Sao Paulo.

Table 1. Secondary beams produced by the RIBRAS and Twinsol.

\begin{tabular}{|c|c|c|}
\hline beam & reaction production & intensity $(\mathrm{ps} / 1 \mu \mathrm{A})$ \\
\hline${ }^{6} \mathrm{He}$ & ${ }^{9} \mathrm{Be}\left({ }^{7} \mathrm{Li},{ }^{6} \mathrm{He}\right)$ & $10^{6}$ \\
${ }^{7} \mathrm{Be}$ & ${ }^{3} \mathrm{He}\left({ }^{6} \mathrm{Li},{ }^{7} \mathrm{Be}\right)$ & $10^{5}$ \\
${ }^{8} \mathrm{Li}$ & ${ }^{9} \mathrm{Be}\left({ }^{7} \mathrm{Li},{ }^{8} \mathrm{Li}\right)$ & $10^{6}$ \\
${ }^{8} \mathrm{~B}$ & ${ }^{3} \mathrm{He}\left({ }^{6} \mathrm{Li},{ }^{8} \mathrm{~B}\right)$ & $5 \times 10^{4}$ \\
${ }^{10} \mathrm{Be}$ & ${ }^{9} \mathrm{Be}\left({ }^{11} \mathrm{~B},{ }^{10} \mathrm{Be}\right)$ & $10^{4}$ \\
& & \\
\hline
\end{tabular}

inant ions out of the bandpass of the second solenoids. An additional cleaning of the secondary beam can be obtained by using time of $\mathrm{f}$ ight (TOF).

For low-energy beams, one-nucleon or few nucleons transfer reactions are the best options to produce radioactive nuclear due to its high cross sections. For instance, the one-nucleon transfer reaction ${ }^{9} \mathrm{Be}\left({ }^{7} \mathrm{Li},{ }^{6} \mathrm{He}\right)$ is used in TwinS ol and RIBRAS to produce reasonably intense ${ }^{6} \mathrm{He}$ beam $\left(10^{6} \mathrm{pps}\right)$ for $1 \mu \mathrm{A}$ of primary beam. In Table- 1 we list the beams produced so far by these two facilities and its respective production reactions and intensity.

\section{Elastic Scattering with low-energy light radioactive ion beams.}

Several experiments to investigate reactions induced by these low-energy light radioactive nuclear beams have been performed at University São Paulo and University of Notre Dame. In particular, the low energy RNBs produced in these labs were very reliable to investigate elastic scattering, transfer and breakup reactions. In general, elastic scattering can be an interesting measurement, specially when one of the interacting particles is a loosely bound nucleus. Some of the unusual features of light weakly-bound nuclei, such as extended halos or neutron skins, can be present in the elastic scattering as inf uence of competing mechanisms, and coupled-channel analysis would be required. Among these mechanisms, direct or sequential breakup can become important, even at relatively low incident energies, and in this case, Continuum Discretized Coupled Channel (CDCC) calculations would be required. The importance of these couplings has been discussed in the investigation of elastic scattering of the neutron-rich nucleus ${ }^{6} \mathrm{He}$ on different targets such as ${ }^{209} \mathrm{Bi}[5],{ }^{120} \mathrm{Sn}$ [7], ${ }^{64} \mathrm{Zn}[8]$ and in light targets such as ${ }^{27} \mathrm{Al}$ [6]. More recently, elastic scattering measurements of ${ }^{8} \mathrm{~B}$ and ${ }^{7} \mathrm{Be}$ on ${ }^{58} \mathrm{Ni}$ have been reported [9]. Their analysis showed that, due to the small binding energy of ${ }^{8}$ B (B.E. $=0.137 \mathrm{MeV}$ ) breakup was the responsible for the increase in the total cross sections. See also ref. [10] for a more detailed discussion on this topic.

As a systematic study of the elastic scattering of light weakly-bound and exotic nuclei, we performed a series of measurements with ${ }^{6} \mathrm{He}$ radioactive beams produced by the RIBRAS system on different targets. Among these measurements we have ${ }^{6} \mathrm{He}+{ }^{27} \mathrm{Al}[6]$, which was the f rst published results using the RIBRAS facility, ${ }^{6} \mathrm{He}+{ }^{51} \mathrm{~V}[11]$, and more recently, ${ }^{6} \mathrm{He}+{ }^{120} \mathrm{Sn} \mathrm{[7]}$ and ${ }^{6} \mathrm{He}+{ }^{9} \mathrm{Be}[12]$. From the analysis of the obtained angular distributions we could observe an enhancement of the elastic scattering cross sections for ${ }^{6} \mathrm{He}$ on heavy targets such as ${ }^{120} \mathrm{Sn}$, which is less extensive for lighter targets such as ${ }^{27} \mathrm{Al}$ and ${ }^{9} \mathrm{Be}$. For ${ }^{6} \mathrm{He}$ scattering in all targets, a better description of the experimental angular distributions is achieved with CDCC calculations considering $2 n-{ }^{4} \mathrm{He}$ and $n-n-{ }^{4} \mathrm{He}$ conf guration for ${ }^{6} \mathrm{He}$ nucleus, f nal 3-body and 4-body, respectively.

Another series of elastic scattering measurements have been performed with radioactive projectiles, ${ }^{8} \mathrm{Li},{ }^{7} \mathrm{Be}$ and ${ }^{10} \mathrm{Be}$, produced also by the RIBRAS facility, on a light target ${ }^{12} \mathrm{C}$. The ${ }^{12} \mathrm{C}$ target has been chosen to investigate possible nuclear effects, which are expected to be more important for a lighter target, rather than the dominant Coulomb effects expected for heavier-mass targets. The elastic scattering angular distributions of ${ }^{8} \mathrm{Li}+{ }^{12} \mathrm{C}$ have been measured at $23.9 \mathrm{MeV}$. The analysis of this angular distribution is presented in ref. [13]. Elastic scattering angular distributions of Beryllium isotopes, ${ }^{7} \mathrm{Be},{ }^{9} \mathrm{Be}$ and ${ }^{10} \mathrm{Be}$, on a natural carbon target were measured at $\mathrm{E}_{l a b}=18.8 \mathrm{MeV}, 26.0$ $\mathrm{MeV}$ and $23.2 \mathrm{MeV}$, respectively. They are displayed in Figs. 2, 3 and 4. The angular distribution for ${ }^{7} \mathrm{Be}$ radioactive ion beam was in part measured at Notre Dame with TwinS ol and in part at São Paulo with RIBRAS . Later, angular distributions were measured for the ${ }^{10} \mathrm{Be}$ radioactive ion beam together with the contaminant ${ }^{9} \mathrm{Be}$ ions, which was simultaneously produced by the RIBRAS system. Details of these particular experiments will be given in the forthcoming paper [14]. Also, in Fig. 5 we show the elastic scattering angular distribution for the ${ }^{8} \mathrm{~B}+{ }^{12} \mathrm{C}$ system. The measurement of this angular distribution was performed at University of Notre Dame using a $25.8 \mathrm{MeV}^{8} \mathrm{~B}$ radioactive beam. A more complete analysis, including $\mathrm{CDCC}$ calculation for this system is reported elsewhere, in ref. [15].

These angular distributions were analyzed in terms conventional optical model (OM) with the code FRESCO [16]. We considered double-folding nuclear Sao Paulo Potential [17], plus Coulomb potentials due to uniform charged spheres. The Sao Paulo Potential (SPP), which has energy dependence and non-locality correction, is given by 
Table 2. Analysis with Sao Paulo Potential.

\begin{tabular}{||c|c|c|c|c||}
\hline System & $N_{R}$ & $N_{I}$ & $\chi_{\text {red }}^{2}$ & $\sigma_{R}[\mathrm{mb}]$ \\
\hline${ }^{7} \mathrm{Be}+{ }^{12} \mathrm{C}$ & 1.00 & 0.78 & 5.75 & 1138 \\
${ }^{7} \mathrm{Be}+{ }^{12} \mathrm{C}$ & 1.20 & 0.57 & 3.42 & 1134 \\
${ }^{9} \mathrm{Be},{ }^{12} \mathrm{C}$ & 1.00 & 0.78 & 5.02 & 1340 \\
${ }^{9} \mathrm{Be}+{ }^{12} \mathrm{C}$ & 1.16 & 1.18 & 3.58 & 1492 \\
${ }^{10} \mathrm{Be}+{ }^{12} \mathrm{C}$ & 1.00 & 0.78 & 1.72 & 1334 \\
${ }^{10} \mathrm{Be}+{ }^{12} \mathrm{C}$ & 0.48 & 0.43 & 0.29 & 1141 \\
${ }^{8} \mathrm{~B}+{ }^{12} \mathrm{C}$ & 1.00 & 0.78 & 3.86 & 1171 \\
${ }^{8} \mathrm{~B}+{ }^{12} \mathrm{C}$ & 1.20 & 0.74 & 2.69 & 1191 \\
\end{tabular}

$$
V_{F}=\int \rho_{1}\left(r_{1}\right) \rho_{2}\left(r_{2}\right) v_{N N}\left(\vec{R}+\overrightarrow{r_{2}}-\overrightarrow{r_{2}}\right) d \overrightarrow{r_{1}} d \overrightarrow{r_{2}}
$$

where $\rho_{1}$ and $\rho_{2}$ are the matter density for the projectile and target, $v_{N N}$ is the effective potential between them given by $v_{N N}(\vec{r}) \simeq V_{0} \delta(\vec{r})$. The average diffuseness for the charge and matter distributions are $a=0.53 \mathrm{fm}$ and $a=0.56$ $\mathrm{fm}$, respectively. The Sao Paulo potential is then used as optical potential with no free parameters,

$$
V_{o p t}=N_{r} V_{F}+i N_{i} V_{F},
$$

here $N_{R}$ and $N_{I}$ stand for normalizations of the real and imaginary part of the potential. The standard values for these parameters are $N_{R}=1.0$ and $N_{I}=0.78$, which were obtained from a large systematic [17]. Using these values, SPP would have no free parameters and can be used as a bare potential. Sometimes a better description of the angular distribution is achieved by considering a different normalization, and, in this case, the anomalous values would be masking important contributions from coupling channels.

We analyzed these angular distributions considering two possibilities: using the standard values for the normalization of the SPP $\left(N_{R}=1.0\right.$ and $\left.N_{I}=0.78\right)$ and with values obtained from a minimum $\chi^{2} \mathrm{ftting}$. In Table- 2 we list the results of this analysis and also the respective total reaction cross section obtained from these analysis. The SPP reproduces quite well the absolute normalization, which is of some interest considering that this folding-model potential has no free parameters. An improvement of the description of the data could be obtained by adjusting the normalizations of the real and imaginary part of the potential, $N_{R}$ and $N_{I}$, respectively. The new normalization did not change considerably the total reaction cross sections. Values different from the standard $\left(N_{R}=1.0\right.$ and $\left.N_{I}=0.78\right)$ would be indication of the importance of other channels. A signif cant reduction of the $N_{R}$ and $N_{I}$ were found to better describe the angular distribution for the ${ }^{10} \mathrm{Be}+{ }^{12} \mathrm{C}$. This is a clear indication of the need of coupled channel calculation for this system. This has been done and the results will be present in the forthcoming paper [14].

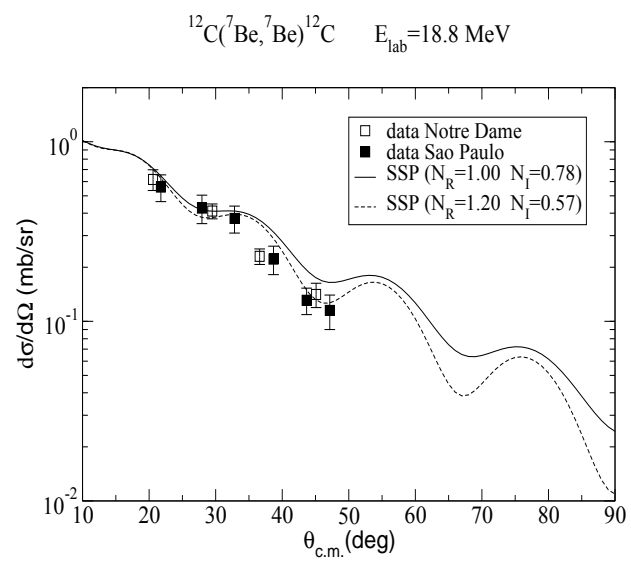

Fig. 2. The differential cross sections for the elastic scattering ${ }^{12} \mathrm{C}\left({ }^{7} \mathrm{Be},{ }^{7} \mathrm{Be}\right){ }^{12} \mathrm{C}$ at $25.8 \mathrm{MeV}$ incident laboratory energy. The curves are optical-model calculations with double-folding SPP.

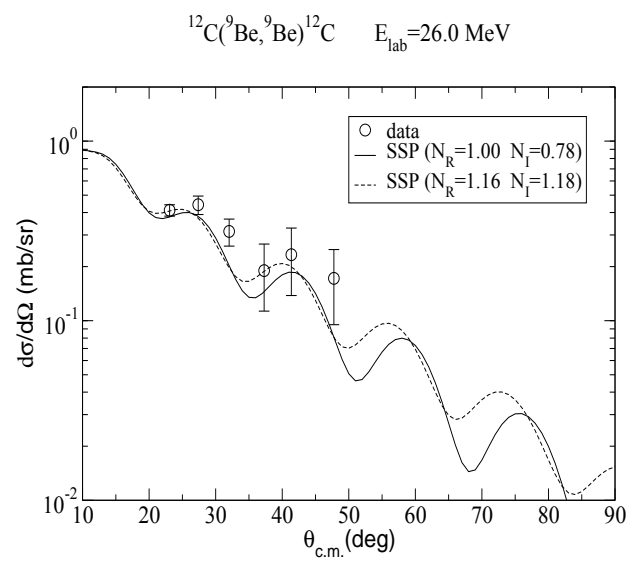

Fig. 3. The differential cross sections for the elastic scattering ${ }^{12} \mathrm{C}\left({ }^{9} \mathrm{Be},{ }^{9} \mathrm{Be}\right){ }^{12} \mathrm{C}$ at $18.8 \mathrm{MeV}$ incident laboratory energy. The curves are optical-model calculations with double-folding SPP.

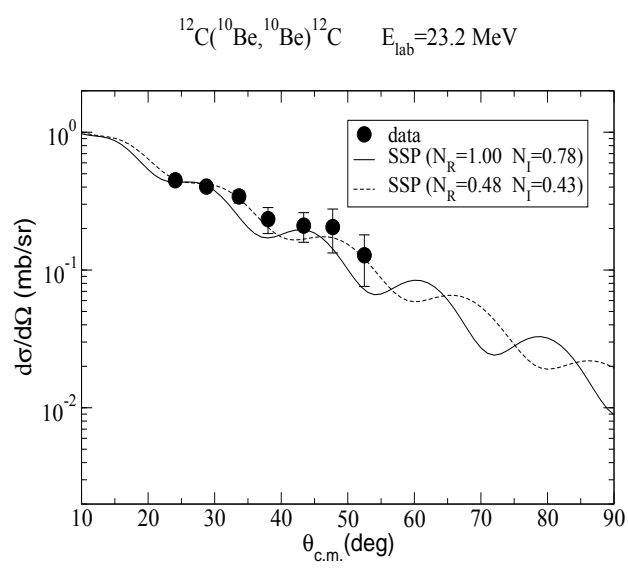

Fig. 4. The differential cross sections for elastic scattering ${ }^{12} \mathrm{C}\left({ }^{10} \mathrm{Be},{ }^{10} \mathrm{Be}\right){ }^{12} \mathrm{C}$ at $12.3 \mathrm{MeV}$ incident laboratory energy. The curves are optical-model calculations with double-folding SPP. 


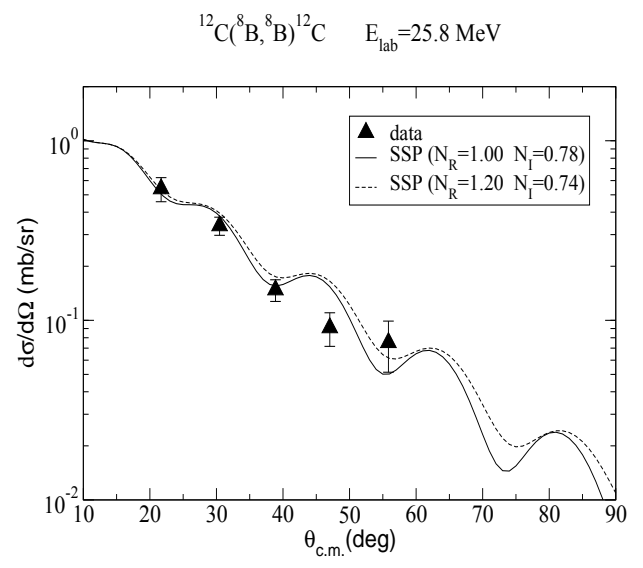

Fig. 5. The differential cross sections for elastic scattering ${ }^{12} \mathrm{C}\left({ }^{8} \mathrm{~B},{ }^{8} \mathrm{~B}\right){ }^{12} \mathrm{C}$ at $25.8 \mathrm{MeV}$ incident laboratory energy. The curves are optical-model calculations with double-folding SPP.

\section{Total reaction cross sections.}

Another important information that can be deduced from the elastic scattering analysis is the total reaction cross sections. In Table- 2 we listed the deduced total reaction cross section for the several systems measured. Comparing total cross sections for different systems can be an interesting way to investigate the inf uence or competition of reaction mechanisms such as breakup, transfer and fusion. In particular, if among these systems we have weakly-bound or exotic nuclei projectile. However, to compare the crosssections for systems with different Coulomb barriers and different geometry, it often is necessary to suppress the differences arising from the size and charges of the systems.

There are different proposals in the literature of how one should re-normalize the total reaction cross section for different systems. Gomes et al. [18] suggested that the cross sections and energies could be re-normalized by

$$
\sigma_{\text {red }}=\frac{\sigma_{R}}{\left(A_{p}^{1 / 3}+A_{t}^{1 / 3}\right)^{2}}
$$

and

$$
E_{\text {red }}=E_{\mathrm{cm}} \frac{\left(A_{p}^{1 / 3}+A_{t}^{1 / 3}\right)}{Z_{p} Z_{t}},
$$

with $Z_{P}\left(Z_{T}\right)$ and $A_{P}\left(A_{T}\right)$ standing for the charge and mass of the projectile (target), respectively, and $\sigma_{R}$ the total reaction cross section. In principle, by considering this reduction, the geometrical effects related to masses of the collision partners are removed. Also, eventual anomalous values of the reduced radius $r_{0}$, which can be related to the physical processes or specif c feature of the projectile nuclear matter density to be investigated, are not washed out.

Analysis using this reduction procedure was performed considering systems with light projectiles, ${ }^{6} \mathrm{Li},{ }^{7} \mathrm{Li},{ }^{9} \mathrm{Be}$, ${ }^{6} \mathrm{He}$ and ${ }^{8} \mathrm{~B}$, on heavy targets such as ${ }^{58} \mathrm{Ni},{ }^{64} \mathrm{Zn}$ and ${ }^{209} \mathrm{Bi}$, where the Coulomb breakup predominates over the nuclear breakup [19]. It is observed that a larger reduced total reaction cross sections are obtained at energies around the Coulomb barrier for exotic projectile $\left({ }^{6} \mathrm{He}\right.$ and $\left.{ }^{8} \mathrm{~B}\right)$ followed by the weakly-bound nuclei $\left({ }^{6} \mathrm{Li},{ }^{7} \mathrm{Li},{ }^{8} \mathrm{Li}\right.$ and $\left.{ }^{9} \mathrm{Be}\right)$ and then the tightly-bound nuclei $\left({ }^{16} \mathrm{O}\right.$ and $\left.{ }^{4} \mathrm{He}\right)$, which produces the smallest total reaction cross section [19]. This behaviour has been explained as to be due to effect of breakup and transfer channels, for ${ }^{8} \mathrm{~B}$ and ${ }^{6} \mathrm{He}$, respectively. Another of such analysis has been performed by Aguilera et al. [20] where he compared total reaction cross sections of available data for ${ }^{6} \mathrm{He}$ and ${ }^{4} \mathrm{He}$ projectile on several targets. The different trends that these systems follow indicates that static and/or dynamics effects may play important role in the total reaction cross sections. According to refs. [20] and [21], dynamics effect would be more important in the region near and below the reduced barrier, and static effects would be relevant for all energies. So, it would be interesting if one could disentangle these effects by considering another way to re-normalize (reduce) the data. Considering this, Canto et al. [22] suggested the use of an universal function $F(x)$, where statics effects arising from the weakly-bound and exotic nuclei would be taken into account by the characteristics of the barrier of each system. This universal function applied to reduce the total reaction cross section is given by:

$$
F(x)=\frac{2 E_{c m}}{\hbar \omega R_{B}^{2}} \sigma_{R}
$$

with

$$
x=\frac{E-V_{B}}{\hbar \omega}
$$

where, $V_{B}, R_{B}$ and $\hbar \omega$ are the height, radius and curvature of the barrier, respectively, and $\sigma_{R}$ is the total reaction cross section.

We applied this reduction procedure to the total reaction cross section obtained for several projectiles, with different binding energies, on ${ }^{12} \mathrm{C}$ target. Angular distributions for ${ }^{6} \mathrm{He}[23-25],{ }^{6} \mathrm{Li}[26-28],{ }^{7} \mathrm{Li} \mathrm{[27],}{ }^{8} \mathrm{Li}$ [13], ${ }^{9} \mathrm{Be}[29],{ }^{11} \mathrm{~B}$ [30] and ${ }^{16} \mathrm{O}$ [31], were re-analyzed with optical model using SPP in the same way as the ones obtained in this work for ${ }^{7} \mathrm{Be},{ }^{9} \mathrm{Be},{ }^{10} \mathrm{Be}$ and ${ }^{8} \mathrm{~B}$. Among these chosen systems we have combinations of weakly-bound projectiles, such as ${ }^{6,7,8} \mathrm{Li},{ }^{7,9} \mathrm{Be}$, exotic projectile as ${ }^{6} \mathrm{He}$ and ${ }^{8} \mathrm{~B}$, and more tightly-bound projectiles such as ${ }^{10} \mathrm{Be}$, ${ }^{16} \mathrm{O}$ and ${ }^{11} \mathrm{~B}$. To obtain the barrier parameters, $R_{B}, V_{B}$ and $\omega$, for each system, necessary for this reduction, we $\mathrm{ft}$ ted a parabola around the barrier to the real part of the SPP potential used to describe the angular distribution for each system. Using these parameters we then deduced the universal function $F(x)$. The obtained universal function $F(x)$ as a function of the parameters $x$ for each system can be seen in Fig. 6. This universal function is derived from Wong's approximation [33], and thus, when valid, is given by:

$$
F_{0}(x)=\frac{2 E}{\hbar \omega_{0} R_{B}^{2}} \sigma_{R}=\ln \left[1+e^{(2 \pi x)}\right]
$$

When Shorto et al. [32] considered this universal function reduction procedure to light projectiles on heavier tar- 


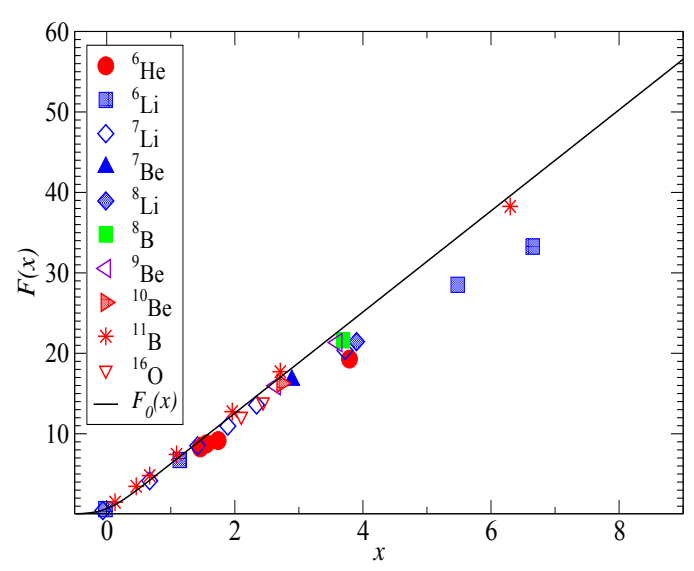

Fig. 6. The reduced reaction cross sections considering the universal function $F(x)$ for lithium isotopes and some other weaklybound and tightly-bound projectiles on ${ }^{12} \mathrm{C}$. The solid line curve is the function $F_{0}(x)=\ln \left[1+e^{(2 \pi x)}\right]$, explained in the text.

gets, such as ${ }^{58} \mathrm{Ni},{ }^{64} \mathrm{Zn}$ or even ${ }^{209} \mathrm{Bi}$, where coulomb breakup predominates over the nuclear breakup [32], larger reduced total reaction cross sections were obtained at energies around the coulomb barrier for exotic nuclei $\left({ }^{6} \mathrm{He}\right.$ and $\left.{ }^{8} \mathrm{~B}\right)$ followed by the weakly bound nuclei $\left({ }^{6} \mathrm{Li},{ }^{7} \mathrm{Li},{ }^{8} \mathrm{Li}\right.$ and $\left.{ }^{9} \mathrm{Be}\right)$ and then for the tightly bound nuclei $\left({ }^{16} \mathrm{O}\right.$ and $\left.{ }^{4} \mathrm{He}\right)$. Similar behavior is observed when we consider the procedure proposed by Gomes et al. [18] to reduce the total reaction cross section. Since static effect due to the neutron skin or halo for the exotic nuclei ${ }^{6} \mathrm{He}$ and ${ }^{8} \mathrm{~B}$ are, in principle, washed out in this universal function procedure, this might be an indication of the importance of dynamic effects. Dynamic effect would related to the role played by transfer and/or breakup channels in the total reaction cross section. However, in our analysis of the total reaction cross section of different projectiles on light ${ }^{12} \mathrm{C}$ target, this behavior is not observed. As one can see in Fig. 6, the $F(x)$ function for weakly-bound lithium and beryllium isotopes, for ${ }^{6} \mathrm{He}$ and ${ }^{8} \mathrm{~B}$ as well as for tightly-bound nuclei as ${ }^{10} \mathrm{Be},{ }^{11} \mathrm{~B}$ and ${ }^{16} \mathrm{O}$ follow the same trend. This may indicate that coupling to other reaction channels may not be as important. A linear plot for the data is adopted in Fig. 6 since most of the data were measured above coulomb barrier. Also, the solid curve in this f gure is the function $F_{0}(x)=\ln \left[1+e^{(2 \pi x)}\right]$. When data, above the coulomb barrier, follows this curve, it implies, in principle, that the reaction cross sections can be described by Wong's approximation.

\section{5 summary}

I reported here on the measurements and analysis of elasticscattering angular distributions for projectiles such as ${ }^{8} \mathrm{~B}$, ${ }^{7} \mathrm{Be},{ }^{9} \mathrm{Be}$ and ${ }^{10} \mathrm{Be}$ on ${ }^{12} \mathrm{C}$ target. These angular distributions are part of a systematic investigation of the elastic scattering of weakly-bound light nuclei being performed with RIBRAS facility at University of Sao Paulo. The measured angular distributions were analyzed with double-folding SPP optical potentials. Also, dynamic and static effects on the total reaction cross section were investigate by comparing the reduced reaction cross sections for system with lithium and beryllium isotopes projectiles as well as for with borromean projectile ${ }^{6} \mathrm{He}$, the proton-halo ${ }^{8} \mathrm{~B}$ and tightlybound ${ }^{11} \mathrm{~B}$ on ${ }^{12} \mathrm{C}$. We found that all these systems follow more or less the same trends in this description, indicating that, for light projectile on light target, the effects of the binding energy through breakup and/or other reaction mechanism on the total reaction cross sections is likely small.

\section{Acknowledgments}

I would like to thank prof. J. J. Kolata and F. Becchetti for their supportive collaboration in the experiments that I have performed at Notre Dame over the past years and for the RIBRAS collaboration people for the help with experiments performed at São Paulo. I also thank CAPES and $\mathrm{CNPq}$ for the f nancial support.

\section{References}

1. R. Lichtenthaler et al., Eur. Phys. J. A 25, S-01, 733 (2005).

2. R. Lichtenthaler et al., Eur. Phys. Journal. Special Topics, 150,27 (2007).

3. F. D. Becchetti et al., Nucl. Instr. and Methods in Res. A 505, 377 (2003).

4. J. T. Huang, C. A. Bertulani, and V. Guimarães.

Atomic Data and Nuclear Data Tables 96, 824-847, (2010).

5. T. Matsumoto, T. Egami, K. Ogata, Y. Iseri, M. Kamimura, M. Yahiro, Phys. Rev. C 73, 051602 (2006).

6. E. A. Benjamim et al.. Physics Letters 647, 30 (2007).

7. P. N. de Faria et al., Phys. Rev. C 81, 044605 (2010).

8. A. Di Pietro et al., Phys. Rev. C 69, 044613 (2004).

9. E. F. Aguilera et al., Phys. Rev. C 79, 021601 (2009).

10. J. Lubian, T. Correa, E. F. Aguilera, L. F.Canto, A. Gomez-Camacho, E. M. Quiroz, P. R. S. Gomes, Phys. Rev. C 79, 064605 (2009).

11. A. Lepine-Szily et al., Nuclear Physics A 834, 491 (2010).

12. K. C. C. Pires et al., 12th International Conference on Nuclear Reaction Mechanisms, 15-19 Jun 2009, Varenna, Italy. CERN-Proceedings-2010-001, pg. 337 (2010). Complete work submitted to PRC.

13. A. Barioni, V. Guimarães et al., Phys. Rev. C 80, 034617 (2009).

14. J. C. Zamora, A. Barioni, V. Guimarães et al., Submitted to Phys. Rev. C (2011).

15. A. Barioni, J. C. Zamora, V. Guimarães et al., Submitted to Phys. Rev. C (2011).

16. I. J. Thompson, Computer Physics Reports, 7, 167 212 (1988). 
17. L. C. Chamon, B. V. Carlson, L. R. Gasques, D. Pereira, C. De conti, M. A. G. Alvarez, M. S. Hussein, M. A. Ribeiro, E. S. Rossi, C. P. Silva, Phys. Rev. C 66, 014610 (2002).

18. P. R. S. Gomes, J. Lubian, I. Padron, R. M. Anjos, Phys. Rev. C 71, 017601 (2005).

19. J. J. Kolata and E. F. Aguilera, Phys. Rev. C 79, 027603 (2009).

20. E. F. Aguilera, I. Martel, A. M. Sanchez-Benitez, L. Acosta. Phys. Rev. C 83021601 (2011).

21. P. R. S. Gomes and L. F. Canto and J. Lubian and M. S. Hussein, Physics Letters B 695, 320 - 323 (2011).

22. L. F. Canto, P. R. S. Gomes, J. Lubian, L. C. Chamon and E. Crema, Journal of Physics G 36, 015109 (2009).

23. R. J. Smith, J. J. Kolata, K. Lamkin, A. Morsad, K. Ashktorab, F. D. Becchetti, J. A. Brown, J. W. Janecke, W. Z. Liu, D. A. Roberts, Phys. Rev. C 43 761-765 (1991).

24. R. E. Warner et al., Phys. Rev. C 51, 178-181 (1995).

25. M. Milin et al., Nuclear Physics A 730, 285 - 298 (2004).

26. J. E. Poling, E. Norbeck, R. R. Carlson, Phys. Rev. C 5, 1819-1825 (1972.

27. J. E. Poling, E. Norbeck, R. R. Carlson, Phys. Rev. C 13, 648 (1976).

28. D. E. Trcka, A. D. Frawley, K. W. Kemper, D. Robson, J. D. Fox, E. F. Myers, Phys. Rev. C 41 (1990).

29. L. Jarczyk, J. Okolowicz, A. Strzalkowski, K. Bodek, M. Hugi, L. Lang, R. Muller, E. Ungrich, Nuclear Physics A 316, 139 (1979).

30. L. Jarczyk, B. Kamys, A. Strzałkowski, A. Szczurek, M. Godlewski, J. Lang, R. Müller, J. Sromicki, Phys. Rev. C 31, 12 (1985).

31. P. Charles, F. Auger, I. Badawy, B. Berthier, M. Dost, J. Gastebois, B. Fernandez, S. M. Lee, E. Plagnol, Physics Letters B 62, 289 (1976).

32. J. M. B. Shorto, P. R. S. Gomes, J. Lubian, L. F. Canto S. Mukherjee and L. C. Chamon, Physics Letters B 678, 77 (2009).

33. C. Y. Wong, Phys. Rev. Lett. 31, 766-769 (1973). 